\title{
CÓMO MEJORAR LA AUTORREGULACIÓN DEL ESTUDIO EN LA EDUCACIÓN SECUNDARIA. VALORACIÓN DE UN PROGRAMA DE ACCIÓN TUTORIAL
}

\author{
Manuel Montanero Fernández \\ Lourdes González Ponte \\ Universidad de Extremadura
}

\begin{abstract}
RESUMEN. En este artículo se analizan las diferentes enfoques de intervención psicopedagógica que se han empleado para potenciar las estrategias de autorregulación del estudio de los alumnos de Secundaria y se valora el efecto de un programa integrador desarrollado desde la tutoría. Los resultados muestran una disminución significativa en las conductas disruptiva registradas por sujetos con problemas de aprendizaje durante la intervención.
\end{abstract}

ABSTRACT. In this paper we analyse different educational intervention approaches usually employed to improve self-regulated learning strategies in Secondary Education. We have assessed the effect of an integrative program developed from tutorship. The results show a meaningful decrease in the negative conduct registered by subjects with learning disabilities during the intervention.

\section{Introducción}

El desarrollo de la capacidad de "aprender a aprender" es una de las tareas más relevantes de la Orientación psicopedagógica (M.E.C., 1990). Esta relevancia se ha traducido particularmente en una amplia divulgación de programas y materiales dirigidos a la instrucción de estrategias de aprendizaje, desde enfoques muy diversos (Repetto, 1990, 1994), que abarcan desde el entrenamiento de estrategias cognitivas y metacognitivas con tareas "independientes de contenido", hasta la enseñanza de estrategias específicas de comprensión de textos, de solución de problemas matemáticos o de regulación de las actividades de estudio

Dentro de este último ámbito, el entrenamiento de estrategias de apoyo (Dansereau, 1979) y la mejora de los hábitos de estudio autónomo constituyen, en concreto, una de las principales demandas que los tutores, los padres o los mismos alumnos plantean a los departamentos de orientación en la Educación Secundaria. Generalmente, el perfil del alumno con una necesidad educativa específica en dicho ámbito no presenta condiciones de discapacidad, empobrecimiento sociocultural o desmoti- 
vación generalizada y manifiesta por el trabajo escolar. Se trata, por el contrario, de estudiantes cuyo bajo rendimiento en diversas áreas curriculares se achaca a problemas de atención y aprovechamiento del tiempo de trabajo, o a lo que ha dado en llamarse "malos hábitos de estudio".

Aunque desde algunos sectores se ha acusado a los nuevos estilos educativos que se han ido imponiendo en el contexto familiar y al propio sistema de promoción escolar como los principales responsables de la proliferación de este problema en la enseñanza obligatoria, lo cierto es que se trata también de un importante campo para una actuación educativa de carácter específico, a coordinar desde los departamentos de orientación. La intervención psicopedagógica, en este sentido, se fundamenta a menudo en el desarrollo de actividades de tutoría poco sistematizadas o en meras entrevistas informativas sobre los factores que favorecen el trabajo intelectual. Sin embargo, al margen de los contenidos de aprendizaje de los que se ocupe, el estudio autónomo constituye una actividad compleja, intensa e intencional en la que están implicados múltiples condicionantes ambientales, procesos motivacionales, cognitivos y metacognitivos. Un buen estudiante debe aprender a fijarse metas realistas y progresivas; debe planificar y organizar su trabajo diario; debe desarrollar estrategias para mantener un nivel de esfuerzo y mejorar su rentabilidad; para evaluar objetivamente los errores y recompensarse con los pequeños avances... Las técnicas de intervención psicopedagógica que se han propuesto para potenciar estas capacidades, tanto desde la acción tutorial, como mediante el asesoramiento familiar o la auto-ayuda, se basan principalmente en la aplicación de procedimientos cognitivos y de modificación de conducta a contextos educativos. A continuación presentamos una revisión de tres grupos de técnicas, que hemos clasificado en función de los objetivos y la metodología que utilizan.

a) Técnicas de control estimular y planificación de intervalos de estudio

Desde antes de comenzar el trabajo, el estudiante debe aprender a desarrollar estrategias "contradictractoras" y de control ambiental de los estímulos que perjudican la atención sostenida y la concentración durante el estudio. Frecuentemente se trata de estímulos visuales o sonoros, dependientes del lugar de trabajo. Muchos estudiantes apenas tienen en cuenta algunas condiciones que minimizan el efecto de dichos estímulos o incluso manifiestan preconcepciones erróneas (como la extendida creencia que estudiar con determinado tipo de música favorece la concentración). Las actividades de información y discusión sobre los factores que facilitan la preparación externa del trabajo es el denominador común de casi todos los métodos de "técnicas de estudio" (véase, por ejemplo, Corzo, 1973; Carreño, 1976 Benedito, 1977; Chico, 1981; Selmes, 1987; Brunet, 1988; Álvarez, 1988; Sáenz, 1988; Cañas y Hernández, 1989; Cuenca, 1989; Pérez Avellaneda, 1989; Serafini, 1991; Vidal, 1990; Salas, 1994; Tierno, 1995; Gómez y otros, 1995; Hernández Pina, 1995; Rotger, 1995). Algunas de las condiciones ambientales que se enseña a identificar y controlar en estos métodos se centran en la elección del lugar de trabajo (que sea siempre el mismo; que la habitación esté ventilada y con una temperatura agradable; que esté bien iluminado, suficientemente tranquilo, alejado de la televisión y sin música...). Otras tienen más bien que ver con la elección y disposición de los materiales (que la mesa sea suficientemente amplia y no muy brillante, bien ordenada y con una estan- 
tería lo más cercana posible; con al menos una lámpara de $60 \mathrm{~W}$ que incida desde el lado izquierdo, si el chico es diestro; con una silla cómoda, pero de respaldo recto y no demasiado blando que permita apoyar los pies en el suelo...).

La preparación psicofísica del alumno es todavía más importante, en este caso para restringir los estímulos negativos de origen interno, como la fatiga o el nerviosismo, y para hacerse más resistentes a la distracción de los externos que no podamos suprimir. Padres y alumnos están también frecuentemente desinformados respecto a los condiciones imprescindibles para ir consolidando hábitos como la importancia de dormir un mínimo de $7 \mathrm{~h}$ seguidas y con un horario fijo; no abusar de las bebidas excitantes o las comidas copiosas, especialmente antes de ponerse a estudiar; los beneficios de una dieta rica y equilibrada y del ejercicio físico para el rendimiento intelectual; la conveniencia de estudiar todos los días y a la misma hora; mantener una postura cómoda pero activa durante el estudio, sin recostarse ni levantarse constantemente...

Casi todos los anteriores materiales aportan además información sobre otros factores psicofísicos, como el efecto intrínseco de la fatiga en el mantenimientos de la atención. Cada estudiante es capaz de alcanzar un determinado nivel de concentración, que podría definirse en función de la calidad del rendimiento intelectual y el tiempo que consigue mantenerse. Estos elementos dependen en gran medida de la edad de los sujetos, pero también del entrenamiento y del uso de estrategias para optimizar los intervalos de trabajo. Para conseguir un elevado nivel de rendimiento durante una sesión de estudio es conveniente comenzar por tareas moderadamente difíciles pero no desmotivantes, para continuar con las más complejas y finalizar el intervalo con aquellas que supongan menos esfuerzo intelectual cuando la fatiga comienza actuar (Brunet, 1988; Pérez Avellaneda, 1989). Un requisito fundamental para este objetivo es que el estudiante analice su capacidad inicial, su "curva de rendimiento" (Gómez y cols., 1995), y planifique estratégicamente el trabajo antes de comenzar cada actividad de estudio. Algunos de los principales elementos que debe tener en cuenta en esta planificación son las asignaturas y tareas que debe realizar; la secuencia idónea, considerando su nivel de dificultad; los materiales que necesita tener preparados (para levantarse lo menos posible durante la sesión); el tiempo que va a invertir en total y en cada actividad (incluyendo tareas de repaso); cuándo y durante cuánto tiempo piensa descansar si el tiempo previsto de estudio es largo. Un considerable parte de la acción tutorial en los institutos de secundaria se dedican a actividades de información, instrucción directa y tareas prácticas sobre la importancia de estos factores y estrategias.

Con una finalidad semejante, aunque desde un enfoque de intervención muy diferente, el entrenamiento en técnicas de reducción de la ansiedad se ha mostrado en ciertos casos eficaz para reducir los estímulos negativos que provienen del propio sujeto y mejorar la disposición física y mental durante el estudio o ante un examen (Salazar, 1995). Se trata de procedimientos relativamente sencillos, basados en la inducción de estados de relajación superficial, el control de la respiración (Labrador y cols., 1993), la imaginación de estímulos relajantes (Campillejo, 1995) o la producción de autoverbalizaciones positivas (Dansereau, 1979), que pueden entrenarse en grupo con un coste de tiempo relativamente bajo. 


\section{b) Técnicas de solución de problemas y concertación}

En nuestra opinión, no obstante, resulta demasiado optimista pensar que la mayoría de los alumnos que no aprovechan los tiempos de estudio personal en la Educación Secundaria pueden mejorar su rendimiento simplemente aprendiendo a controlar los estímulos que les distraen. Limitarse a este tipo de intervención, como muchas veces ocurre, supone, por un lado, un excesivo énfasis sobre el "no hacer" o el "no distraerse" como llave de la autorregulación del estudio. Por otro lado, desatiende otros importantes factores cognitivos y emocionales.

En estos últimos años se ha puesto particularmente de manifiesto el papel que el desarrollo emocional ejerce en el rendimiento académico (Goleman, 1996; López, 1999). Una adecuada autoestima y autoeficacia, unas expectativas adaptadas de forma realista a sus auténticas posibilidades, sin pensamientos derrotistas, el reconocimiento del valor del esfuerzo, son elementos muy importantes para que el estudiante se sienta motivado y satisfecho personalmente en su trabajo, aunque a veces se obtengan algunos fracasos. Frecuentemente la dificultad para desarrollar hábitos de estudio está relacionada con la presencia en el adolescente de todo un sistema de motivaciones incompatible con las metas escolares. Éstas tienen esencialmente un carácter "a largo plazo" y apenas generan recompensas materiales; de modo que, si el sujeto no desarrolla también una satisfacción intrínseca con la realización de las tareas de aprendizaje y con el sentimientos de competencia que pueden proporcionar, es muy difícil que consiga movilizar los recursos y el considerable esfuerzo que el éxito académico demanda (Alonso Tapia, 1991).

Por esta y otras razones, cada día se hace más evidente el reto de situar la mejora de los hábitos de estudio en el marco de una intervención psicopedagógica más amplia que abarque también factores socioculturales del contexto escolar y familiar. El diálogo y la reflexión compartida sobre los problemas que el estudiante debe afrontar constituye, en este sentido, el objetivo prioritario a trabajar con padres y tutores. Frente a algunos enfoques educativos exclusivamente "voluntaristas", centrados en "convencer" al alumno de los beneficios a largo plazo, el requisito previo es que éste perciba el estudio como un proyecto "compartido" con sus educadores, principalmente con la familia. Unos y otros deberían consensuar los objetivos y afrontar diariamente las responsabilidades y sacrificios que conlleva el estudio (no sólo inmediatamente antes de un examen o cuando se entregan las notas). Los discursos moralizantes suelen resultar poco eficaces. En muchos casos la efectividad no dependerá tanto de nuestras buenas intenciones como del diseño de actuaciones concretas que involucren auténticamente tanto a los estudiantes como a las familias.

En esta línea, las técnicas de solución de problemas y concertación pueden proporcionar al orientador o al tutor una herramienta útil para potenciar ese contexto de reflexión y compromiso, en dos escalones básicos. En primer lugar, pueden facilitar marcos explícitos de diálogo, que estimulen la reflexión del estudiante y la toma de consciencia sobre las metas y los medios que se necesitan para alcanzarlas. Algunas actividades prácticas y dinámicas de grupo se adaptan particularmente bien, tanto a las tutorías grupales, como al trabajo individual con padres y alumnos. Así, por ejemplo, una sencilla representación gráfica de la distribución del tiempo durante las 24 horas de un día normal y de un día "ideal" desde el punto de vista del estudiante, pueden enriquecer la puesta en 
común posterior sobre los problemas actuales y las necesidades de cambio que cada uno se plantea. Otras dinámicas basados en la solución de problemas (como la correspondiente al material que presentamos en la tabla 1) facilitan más explícitamente que el sujeto reflexione sobre metas específicas y obstáculos para conseguirlas.

\begin{tabular}{||c|l|l|l|l|l||}
\hline $\begin{array}{c}\text { SOLUCIÓN DE } \\
\text { PROBLEMAS }\end{array}$ & ¿Qué hago? & $\begin{array}{c}\text { ¿Qué quiero } \\
\text { hacer? }\end{array}$ & $\begin{array}{c}\text { ¿Cómo lo } \\
\text { hago? }\end{array}$ & $\begin{array}{c}\text { ¿Qué riesgos } \\
\text { puedo encontrar? }\end{array}$ & $\begin{array}{c}\text { iA qué me puedo } \\
\text { comprometer? }\end{array}$ \\
\hline En el instituto & & & & & \\
\hline $\begin{array}{c}\text { En casa, durante } \\
\text { la semana }\end{array}$ & & & & & \\
\hline $\begin{array}{c}\text { Durante el fin } \\
\text { de semana }\end{array}$ & & & & & \\
\hline $\begin{array}{c}\text { Durante las } \\
\text { vacaciones }\end{array}$ & & & & & \\
\hline
\end{tabular}

Tabla 1. Tabla para una actividad de solución de problemas

En un segundo momento, este proceso debería culminar en un compromiso voluntario con objetivos parciales, a corto o a medio plazo. Como ya hemos comentado, en ocasiones, este componente sólo es útil cuando se implica a los educadores, por ejemplo a través de un contrato conductual donde se negocien también los compromisos que están dispuestos a asumir también los padres en el marco de ese proyecto común. Por supuesto que muchos padres no podrán ofrecer a sus hijos todas las condiciones de estudio descritas anteriormente. Pero siempre es posible establecer a ciertas horas algún lugar apropiado, aunque esto suponga algunos sacrificios, como apagar la televisión si se encuentra demasiado cerca... Los compromisos adquiridos deben operativizarse lo más posible en objetivos y tareas específicas, que supongan una progresiva mejora de las actividades de estudio, así como el tipo de recompensa o de compensación por el cumplimiento de los mismos.

\section{c) Técnicas de autocontrol}

Independientemente de la importancia de reflexionar y aprender a comprometerse con las responsabilidades que uno se plantea, la capacidad de autorregulación conlleva además capacidades más específicas de planificación de metas progresivas en relación a la calidad o la cantidad de las conductas, cuya consecución el sujeto tiene que aprender a controlar y reforzar diariamente hasta consolidar un hábito. Este es el objetivo principal de las técnicas de autocontrol, que se pretende conseguir entrenando a los sujetos en el uso de estrategias para alterar la probabilidad de ocurrencia de las conductas conflictivas, en este caso, la distracción o el abandono de la actividad de estudio (Díaz García y cols., 1993). Estas estrategias se basan principalmente en la autoobservación, la programación y el autorrefuerzo de las conductas reguladas por el propio sujeto, mediante el uso de autorregistros. 
En la tabla 2 presentamos un ejemplo de autorregistro que facilita tanto la programación de intervalos de estudio como la autoobservación de las conductas disruptivas. En la columna de la izquierda el alumno debe reflejar los componentes de la planificación diaria de la actividad de estudio (que, a su vez, deriva de una programación previa y progresiva de la distribución semanal del tiempo). En la columna de la derecha debe registrar el número y tipo de conductas negativas, antes y durante la intervención.

La utilidad de la columna de autoobservación, con algunos estudiantes, tiene una doble justificación. Por un lado, los problemas de concentración están frecuentemente relacionados con "autorreacciones cognitivas", que no suelen ser accesibles a la observación externa (Díaz García y cols. , 1993). El cómputo de la frecuencia de "distracciones" anotadas con una cruz en cada sesión de estudio proporciona un modo alternativo de evaluación, cuya información puede además representarse gráficamente en un sencillo eje de coordenadas, con objeto de inferir la evolución global de las conductas y adaptar la programación en función de los progresos del sujetos. Se trata de una evaluación no más subjetiva que la procedente de los tradicionales autoinformes e inventarios sobre autorregulación del estudio. Algunos de los más utilizados en nuestro entorno son el IHE (Pozar, 1972), IME (Baeza, 1979), LASSI (Weinstein y cols., 1983), ACRA (Román y Gallego, 1994) o el IDEA (Vizcarro, 1996). En todos ellos se puede constatar una marcada amenaza de sesgos procedentes la propia percepción (sobre conductas relativamente complejas y remotas en el tiempo) o distorsionadas por la "deseabilidad social".

Por otro lado, el autorregistro puede incrementar el control, no sólo del inicio, sino sobre todo de la terminación de las conductas negativas. El problema de muchos adolescentes no es tanto que se distraigan a menudo cuanto la duración de las distracciones. En los casos más problemáticos, éstas llegan a convertirse en auténticas "ensoñaciones", generadas por asociaciones de pensamientos que el sujeto no interrumpe. El compromiso de registrar cada nueva asociación (sin preocuparse por si uno se vuelve o no a distraer) resulta a la postre incompatible con la ausencia de control que caracterizan a este tipo de distracciones. Lo único que se le pide al estudiante es que cada vez que se percate de que está distraído, se detenga en seco para coger el bolígrafo, acercar la plantilla y marcar una cruz en la casilla correspondiente. Si un instante después vuelve a ocurrirle, repite el proceso tantas veces como sea necesario. De esta forma, podríamos decir que el autorregistro proporciona también un método de detención de los pensamientos negativos para la concentración.

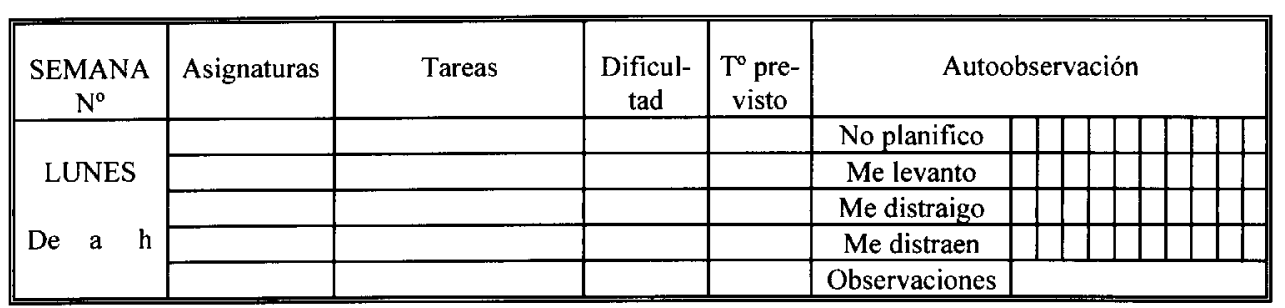

Tabla 2. Autorregistro semanal para la programación de intervalos de estudio (adaptado de Montanero, 1998) 
Finalmente, hemos comprobado que el ejercicio de cuantificación semanal del progreso y su representación gráfica puede ser intrínsecamente reforzante para muchos sujetos. De todas formas, su principal ventaja proviene de su potencialidad para facilitar un diálogo constructivo con padres o tutores, de manera que estos puedan supervisar la progresión del alumno, comprobando que compensa sus fallos y recaídas con un esfuerzo suplementario, y premiando también de muy diversas formas cada uno de sus avances. En otros casos, puede ser necesario negociar determinadas recompensas por el cumplimiento de los objetivos parciales que se habían programado.

En la tabla 3, presentamos un esquema de las diferentes técnicas expuestas hasta ahora, así como los objetivos específicos que pretenden.

\begin{tabular}{|cc|}
\hline TÉCNICAS & OBJETIVOS \\
\hline Instrucción directa y relajación & Control estimular externo \\
\cline { 2 - 2 } & Control estimular interno \\
\hline $\begin{array}{c}\text { Solución de problemas y } \\
\text { concertación }\end{array}$ & Reflexión \\
\hline Autocontrol & Compromiso con metas parciales \\
\cline { 2 - 2 } & $\begin{array}{c}\text { Programación de actividades de estudio } \\
\text { Autoobservación }\end{array}$ \\
\cline { 2 - 2 } & Autorrefuerzo de hábitos \\
\hline
\end{tabular}

Tabla3. Técnicas y objetivos de la intervención psicopedagógica

Las actuaciones de los tutores y orientadores con respecto a la potenciación de la capacidad de autorregular el estudio suele centrarse en la aplicación más o menos aislada de alguno de estos procedimientos (casi siempre limitadas a la preparación de las condiciones ambientales). La finalidad de este trabajo es precisamente valorar la eficacia de un programa de intervención que sistematice y combine estas diferentes técnicas de intervención desde el contexto de la tutoría. Nuestra hipótesis es que la complementación de técnicas de control estimular, solución de problemas y autocontrol producirá mejoras significativas en el rendimiento de los intervalos de estudio de los sujetos sometidos al programa.

\section{Método}

En la investigación participaron 9 alumnos varones de entre 14 y 15 años que fueron seleccionados por un procedimiento no-aleatorio de entre un grupo de 35 alumnos de 3 o de la E.S.O. pertenecientes a un centro privado de Salamanca. Los criterios de selección fueron básicamente dos: la presencia de un bajo rendimiento escolar (tres o más áreas con calificaciones inferiores a 5 en la primera evaluación) y la apreciación por parte del tutor de un manifiesto interés en mejorar los hábitos de estudio y participar voluntariamente en el programa. 
Las actividades del programa de intervención psicopedagógica fueron desarrolladas por el tutor en dos fases. La primera fase abarcó tres semanas en total, en las que se desarrollaron los principales componentes de intervención. Por un lado, los 35 alumnos del grupo completo trabajaron, mediante instrucción directa y dinámicas de grupo, los tres primeros objetivos de la tabla 3, en tres sesiones de tutoría de unos 45 minutos cada una. Por otro lado, se convocó además a los padres a una actividad grupal (de una hora y media de duración) en el que se analizaron también los factores ambientales y motivacionales que intervienen en el rendimiento escolar. Finalmente, los 9 alumnos seleccionado fueron entrevistados dos veces por el tutor (en el intervalo de los diez primeros días de la intervención). Durante una hora y media, aproximadamente, se aplicaron técnicas de concertación de compromisos de mejora, se negoció un contrato conductual y se instruyó a los 9 sujetos sobre la utilización de la hoja semanal de programación y autorregistro.

La segunda fase se prolongó durante 8 semanas más, únicamente con los 9 alumnos seleccionados, que continuaron aplicando los procedimientos de autocontrol ensayados. Cada estudiante debía programar y observar su actividad de estudio en intervalos de unas dos horas (con la presencia en el aula de otros compañeros y del propio tutor). Al comenzar cada semana, debían además asistir a una entrevista de seguimiento individual, de unos 10 minutos de duración. El contenido fundamental de esta entrevista era la cuantificación, y representación en el gráfico personal del alumno de las conductas disruptivas de la semana anterior, cuyas posibles causas y evolución se comentaban.

Como criterio de valoración se tomó la evolución de la frecuencia de esas conductas disruptivas autorregistradas por cada alumno en la columna de autoobservación (ver tabla 2) hasta la finalización del trimestre.

\section{Resultados y discusión}

En al figura 1, se representa la media de conductas negativas, registradas por los propios sujetos a lo largo del trimestre. La mayoría de los alumnos obtiene un resultado semejante a la anterior gráfica: con un primer repunte (debido a las distracciones que produce inicialmente el efecto novedoso de la técnica), que baja bruscamente en las siguientes semanas, para estabilizarse ligeramente por encima, una vez adquirido un cierto hábito. Inicialmente, la mayoría de los alumnos manifestaron, pues, una sensible reducción de conductas disruptivas a partir del segundo mes del programa.

La escaso número de individuos que participaron en el experimento imposibilitaba confirmar los supuestos necesarios para un análisis estadístico según un modelo lineal de medidas repetidas. En su lugar se optó por dos cálculos más simples. Por un lado, se comparó la media de distracciones en las tres primeras semanas (110.29) con las tres últimas (70.03), confirmándose la significatividad de dicha diferencia en la prueba de Wilcoxon $(Z=-2.31 ; p=0.02)$. Por otro lado, se calculó la recta de regresión de todos los registros obtenidos semanalmente por cada sujeto, tomando como variable independiente el factor tiempo. Aunque los registros de 2 de los 9 alumnos no manifestaban una pendiente clara en el gráfico de dispersión, el coeficiente de regresión mostró una disminución significativa de las puntuaciones en la variable "distrac- 


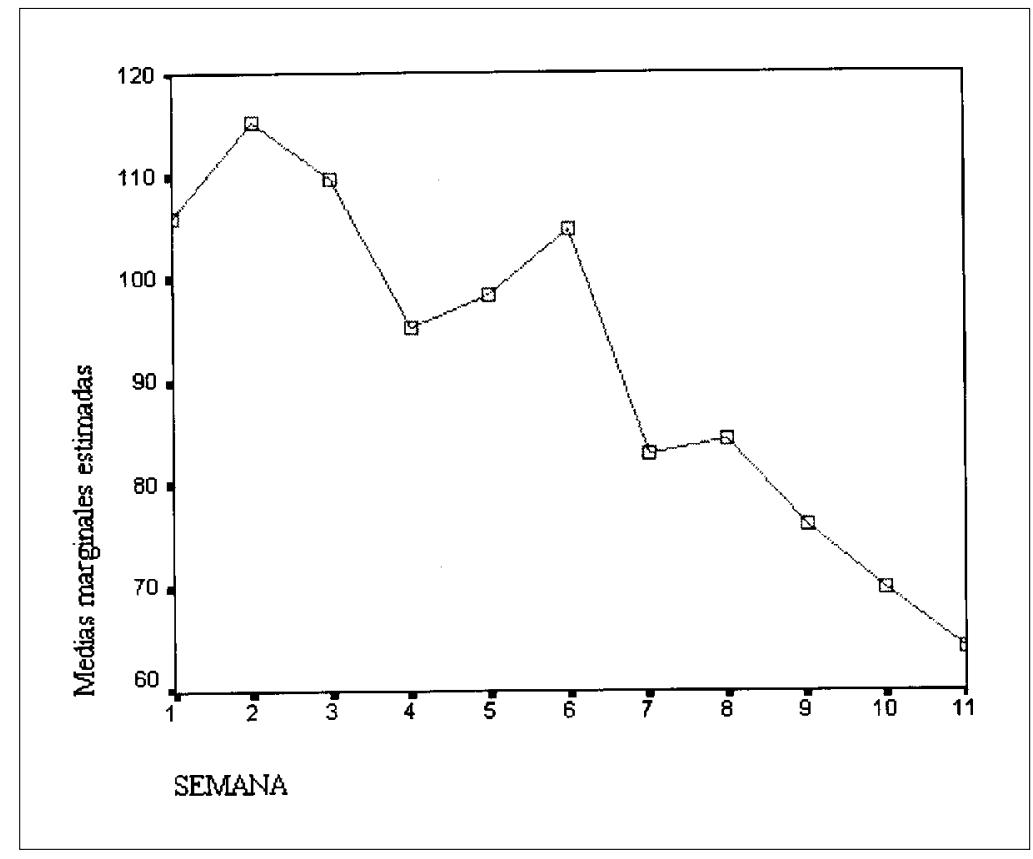

Figura 1. Media de conductas disruptivas registradas en el grupo durante la intervención

ciones", conforme se incrementaban las unidades de la variable "tiempo" $(\mathrm{t}=10.87$; $\mathrm{p}<0.01$ ). En consecuencia, los datos confirman la eficacia general del programa, al menos en cuando al control que los sujetos consiguieron sobre las conductas disruptivas en el estudio.

No obstante, esta investigación presenta evidentes limitaciones que es necesario tener en cuenta en próximos trabajos. En primer lugar, la evaluación de las capacidades de autorregulación del estudio ofrece una indudable complejidad, más allá de la mera cuantificación de conductas disruptivas. Ya hemos comentado que la autoobservación plantea problemas de validez que no se resuelven con los tradicionales autoinformes, sino más bien con triangulaciones entre estos y otros procedimientos de evaluación externa. En nuestro caso, las garantías de sinceridad de los autorregistros se basaban, por un lado, en la voluntariedad de los sujetos y, por otro, en que se aplicaron en tiempos de estudio "vigilados" por el propio tutor (que podía fácilmente comprobar si los alumnos utilizaban realmente el registro). Como contrapartida, esta supervisión pone en duda las generalización de los hábitos de autocontrol a otras situaciones de estudio, sin la presencia física del tutor.

En segundo lugar, las condiciones en que se desarrolló la investigación dificultaban la configuración de un grupo de control, así como un seguimiento más prolongado de los sujetos que participaron en el programa. Lo cual, teniendo en cuenta las características del mismo, supone una amenaza de que las mejoras pudieran estar contaminadas por un efecto "Hawthorne". 
Finalmente, la complejidad de la intervención y la ausencia de otros grupos experimentales imposibilita ciertamente averiguar si dichas mejoras fueron debidas a alguna de las técnicas en particular o a la conjunción de todas ellas.

Aunque esperamos contrastar algunas estas cuestiones en futuras investigaciones, los resultados apoyan al menos la posibilidad de incidir sobre los factores cognitivos, emocionales y comportamentales, implicados en el desarrollo de las capacidades de autorregulación. Frente al diseño de actividades puntuales de uno u otro tipo, los departamentos de orientación deberían promover actuaciones más sistematizadas y diversificadas, en función de las necesidades específicas de los alumnos.

\section{Referencias bibliográficas}

ALONSO TAPIA, J. (1991). Motivación y aprendizaje en el aula. Madrid: Santillana.

ÁlVAREZ, M. (1988). Métodos de estudio. Barcelona: Martínez Roca.

BAEZA, M. (1979). Inventario de métodos de estudio. Madrid: Miñón.

BENEDITO, V. (1977). Métodos de estudio. Barcelona: Círculo editor universo.

BRUNET, J. J. (1987) Cómo programar las técnicas de estudio en EGB. Madrid: San pío X.

CAÑAS, J.L. y HERNÁNDEZ, T. M. (1989). Ayudar a autoestudiar. Madrid: Narcea.

CARREÑO, P.A. (1976). Estudiar, distinto de aburrirse. Entrenamiento para el estudio. Madrid: Rialp.

CHICO, P. (1981). Estudiar con eficacia. Bujedo: La Salle.

CORZO, J.M. (1973). Técnicas de trabajo intelectual. Madrid: Anaya.

CUENCA F. (1989). Cómo estudiar con eficacia. Madrid: Escuela española.

DANSERAU, D. F. (1979). Development and evaluation of learning strategy training program. Journal of education psychology, 71, 64-73.

DíAZ, M.; COMECHE, M.I; VALLEjO, M.A. (1993). Técnicas de autocontrol. En F. J. Labrador, J. A. Cruzado y M. Muñoz, (coord.), Manual de técnicas de modificación y terapia de conducta (pp. 577-593). Madrid: Pirámide.

GOLEMAN, D. (1996). Inteligencia emocional. Barcelona: Kairós.

GÓMEZ, P.C.; GARCÍA, A. y ALONSO, P. (1995). Programas de Técnicas de Trabajo Intelectual. Manual del profesor. Madrid: EOS.

LABRADOR, F.; DE LA PUENTE, M. L. y CRESPO, M. (1993). Técnicas de control de la activación: relajación y respiración. En F. J. Labrador, J. A. Cruzado y Muñoz, M. (coord.), Manual de técnicas de modificación y terapia de conducta (pp. 367-395). Madrid: Pirámide.

LÓPEZ, F. (1999). Problemas afectivos y de conducta en el aula. En C. Coll, A. Marchesi y J. Palacios (comps.). Desarrollo psicológico y educación (pp. 161-182). Madrid: Alianza.

M.E.C. (1990). La Orientación Educativa y la Intervención Psicopedagógica. Madrid: Dirección General de Renovación pedagógica. 
MONTANERO, M. (1998). La mejora de las habilidades intelectuales en Secundaria. En F. Blázquez, T. González y M. Montanero (1998). Formación psicopedagógica del profesorado de Secundaria (pp. 345-368). Badajoz: I.C.E. de la UEX.

PÉREZ AVELLANEDA, M. (1989). Enseñar a estudiar. Madrid: Escuela Española.

POZAR, F. F. (1972). Inventario de hábitos de estudio. Madrid: TEA.

REPETTO, E. (1990). Entrenamiento metacognitivo, la modificabilidad cognitiva y su transferencia a la comprensión lectora, a la resolución de problemas y al aprendizaje. Revista de Investigación Educativa, 8, 563-587.

REPETTO, E. (1994). Orientación educativa e intervención psicopedagógica. Madrid: UNED.

ROMÁN, J.M. y GALLEGO, S. (1994). ACRA. Madrid: TEA.

ROTGER, B. (1995). Las técnicas de estudio en los programas escolares. Madrid: Cincel-Kaplelusk.

SÁENZ, O. (1988). Técnicas de trabajo Intelectual. En Sáenz, O. (dir.), Didáctica General (pp. 165-214). Madrid: Anaya.

SALAS, M. (1994). Técnicas de estudio. Madrid: Ediciones del Prado.

SALAZAR, J. I. (1995). Relajación y concentración para mejorar en el estudio. Zizur: Haranegi.

SELMES, I. (1987). La mejora de las habilidades para el estudio. Madrid: Paidós.

SERAFINI, M. T. (1991). Cómo se estudia. Barcelona: Paidós.

TIERNO, B. (1995). Las mejores técnicas de estudio. Madrid: Planeta.

VIDAL, V. (1990). Manual para la confección de programa de desarrollo individual. Técnicas de trabajo Intelectual (volumen 3). Madrid: EOS.

VIZCARRO, C. (1996). Development of an inventory to mesure learning strategies. En M. Birembaum y F. Dochy (eds.), Alternatives in assessment of achivements learning processes and prior knowledge. Boston: Kluwer Academic Press.

WEINSTEIN, C.; GOEZTS, E. T. y ALEXANDER, P. A. (1983). Learning and study strategies, issues in assessment, instruction and evaluation. New York: Academic Press. 